\title{
Monte Carlo Glauber model with meson cloud: predictions for 5.44 TeV Xe + Xe collisions
}

\author{
B. G. Zakharov ${ }^{\mathrm{a}}$ \\ L.D. Landau Institute for Theoretical Physics, GSP-1, 117940, Kosygina Str. 2, 117334 Moscow, Russia
}

Received: 15 April 2018 / Accepted: 19 May 2018 / Published online: 29 May 2018

(c) The Author(s) 2018

\begin{abstract}
We study, within the Monte-Carlo Glauber model, centrality dependence of the midrapidity charged multiplicity density $d N_{c h} / d \eta$ and of the anisotropy coefficients $\varepsilon_{2,3}$ in $\mathrm{Pb}$ $+\mathrm{Pb}$ collisions at $\sqrt{s}=5.02 \mathrm{TeV}$ and in $\mathrm{Xe}+\mathrm{Xe}$ collisions at $\sqrt{s}=5.44 \mathrm{TeV}$. Calculations are performed for versions with and without nucleon meson cloud. The fraction of the binary collisions, $\alpha$, has been fitted to the data on $d N_{c h} / d \eta$ in $\mathrm{Pb}+\mathrm{Pb}$ collisions. We obtain $\alpha \approx 0.09(0.13)$ with (without) meson cloud. The effect of meson cloud on the $d N_{c h} / d \eta$ is relatively small. For Xe + Xe collisions for $0-5 \%$ centrality bin we obtain $d N_{c h} / d \eta \approx 1149$ and 1134 with and without meson cloud, respectively. We obtain $\varepsilon_{2}(\mathrm{Xe}) / \varepsilon_{2}(\mathrm{~Pb}) \sim 1.45$ for most central collisions, and $\varepsilon_{2}(\mathrm{Xe}) / \varepsilon_{2}(\mathrm{~Pb})$ close to unity at $c \gtrsim 20 \%$. We find a noticeable increase of the eccentricity in $\mathrm{Xe}+\mathrm{Xe}$ collisions at small centralities due to the prolate shape of the Xe nucleus. The triangularity in $\mathrm{Xe}+\mathrm{Xe}$ collisions is bigger than in $\mathrm{Pb}+\mathrm{Pb}$ collisions at $c \lesssim 70 \%$. We obtain $\varepsilon_{3}(\mathrm{Xe}) / \varepsilon_{3}(\mathrm{~Pb}) \sim 1.3$ at $c \lesssim 1 \%$.
\end{abstract}

\section{Introduction}

It is believed that production of soft particles in $A A$ collisions at RHIC and LHC energies occurs via formation of the quark-gluon plasma (QGP) that expands hydrodynamically as a near-ideal liquid [1,2]. Hydrodynamic models have been successfully used for description of the data from RHIC and LHC on centrality dependence of hadron multiplicities and flow effects in $A A$ collisions. The currently available LHC data on $A A$ collisions have been obtained for $\mathrm{Pb}+\mathrm{Pb}$ collisions at $\sqrt{s}=2.76$ and $5.02 \mathrm{TeV}$. Recently at the LHC there has been performed a run for Xe + Xe collisions $(A=129)$ at $\sqrt{s}=5.44 \mathrm{TeV}$. The data from this run will allow to study variation of the $A$-dependence of soft hadron production in $A A$ collisions. One can expect that the flow effects for $\mathrm{Xe}+\mathrm{Xe}$ collisions should be stronger than for $\mathrm{Pb}+\mathrm{Pb}$

a e-mail: bgz@itp.ac.ru collisions because fluctuations in the initial entropy density should increase for nuclei with a smaller nucleon number. For this reason the data on $\mathrm{Xe}+\mathrm{Xe}$ collisions are of great interest for testing the hydrodynamic picture of the QGP fireball evolution. The $\mathrm{Xe}+\mathrm{Xe}$ collisions at $\sqrt{s}=5.44 \mathrm{TeV}$ have been discussed recently in Refs. [3,4].

The hydrodynamic simulations of $A A$ collisions require imposing the initial conditions for the entropy/energy distribution at the QGP production time $\tau_{0} \sim 0.5-1 \mathrm{fm}[5,6]$. One of the popular approach for setting the initial conditions for the QGP fireball in AA collisions is the Monte-Carlo Glauber (MCG) wounded nucleon model [7-10]. In Refs. [11,12] we have developed a version of the MCG wounded nucleon model for nucleons with meson cloud. The meson-baryon Fock components of the nucleon play an important role in the flavor dependence of nucleon parton distribution functions (PDFs) in deep inelastic scattering [13], and allow to explain the violation of the Gottfried sum rule [13]. It is important that, similarly to the wounded nucleon model with constituent quarks [14-19], the meson degrees of freedom lead to a nonlinear increase of $d N_{c h}(A A) / d \eta$ with the number of the wounded nucleons [12]. This effect should emerge independently of the specific mechanism of inelastic interactions. It is important that, contrary to the MCG models with the quark subnucleon degrees of freedom, the interaction of the meson components is better understood, say, within the quark-gluon string model [20,21]. Similarly to the ordinary two-component MCG model without meson cloud [9] the model $[11,12]$ accounts for the contributions from soft interaction (participant wounded nucleons) and from hard binary collisions [22]. However, the results of Refs. $[11,12]$ show that in the presence of the meson-baryon Fock components the required fraction of the binary collisions, $\alpha$, becomes smaller. The results of Ref. [12] show that the meson cloud may improve somewhat agreement with the data on the dependence of the elliptic flow on the charged multiplicity for very small centralities defined via the ZDCs signals for col- 
lisions of the deformed uranium nuclei at $\sqrt{s}=0.193 \mathrm{TeV}$ [23]. In the present paper we apply the model of Refs. [11,12] to obtain predictions for $\mathrm{Xe}+\mathrm{Xe}$ collisions at $\sqrt{s}=5.44$ $\mathrm{TeV}$. To fix the parameters of the model we use the data on $p p$ and $\mathrm{Pb}+\mathrm{Pb}$ collisions.

The plan of the paper is as follows. In Sect. 2 we outline the theoretical framework. In Sect. 3 we present the numerical results. We give conclusions in Sect. 4.

\section{Outline of the model}

In this section we briefly sketch our MCG scheme. We refer the reader to Ref. [12] for more details.

We represent the physical nucleon wave function $\left|N_{\text {phys }}\right\rangle$ in the infinite-momentum frame (IFM) as the Fock-state composition of a bare nucleon $|N\rangle$ and an effective two-body meson-baryon $|M B\rangle$ state

$\left|N_{\text {phys }}\right\rangle=\sqrt{1-n_{M B}}|N\rangle+\int d x d \mathbf{k} \Psi_{M B}(x, \mathbf{k})|M B\rangle$,

where $x$ is the fractional longitudinal meson momentum in the physical nucleon, $\mathbf{k}$ is the transverse meson momentum, $\Psi_{M B}$ is the IMF (light-cone) wave function of the $M B$ Fock state, and

$n_{M B}=\int d x d \mathbf{k}\left|\Psi_{M B}(x, \mathbf{k})\right|^{2}$

is the total weight of the $M B$ Fock state. The previous analyses of the meson cloud effects in deep inelastic scattering (for a review, see Ref. [13]) show that the total weight of the meson-baryon Fock states in the nucleon is $\sim 40 \%$. Due to this, we take for the probability of the effective $M B$ state $n_{M B}=0.4$. Since the meson-baryon component is dominated by the pion-nucleon $\pi N$ state [13], we calculate the IMF distribution of the effective $M B$ state for the $\gamma_{5}$ spin vertex. As in the analyses of the deep inelastic scattering [13], we introduce a phenomenological vertex formfactor, $F$, to account for the internal structure of the hadrons. We take it in the form [13]

$F=\left(\frac{\Lambda^{2}+m_{N}^{2}}{\Lambda^{2}+M_{\pi N}^{2}}\right)^{2}$

where $M_{\pi N}$ is the invariant mass of the $\pi N$ system. We take $\Lambda=1.3 \mathrm{GeV}$, supported by the analysis [24] of the data on the process $p p \rightarrow n X$. This value, at the same time, is also supported by the data on the violation of the Gottfried sum rule for the nucleon PDFs [13]. Note, however, that our results are not very sensitive to the value of $\Lambda$.

In our model, as in the well known version of the MCG wounded nucleon model at the elementary nucleon-level
GLISSANDO [9], the entropy is deposited in the soft sources from participants (related to soft interactions) and in the hard sources from the binary collisions, related to hard reactions, between the colliding particles. However, in our scheme the inelastic interaction of the physical nucleons from the colliding objects may occur as $N+N, N+M B, M B+N$ and $M B+M B$ collisions. Because at high energy the midrapidity multiplicity density for all baryons and mesons should be similar, we assume that the constituents $M$ and $B$ in the $M B$ effective Fock state interact like a pion and a nucleon, respectively. We assume that the inelastic cross sections for the bare baryon and meson states obey the constituent quark counting rule $4 \sigma_{i n}^{N N}=6 \sigma_{i n}^{M B}=9 \sigma_{i n}^{M M}$. We use the Gaussian impact parameter profile for the probability of $a b$ inelastic interaction of the bare constituents

$P_{a b}(\rho)=\exp \left(-\pi \rho^{2} / \sigma_{i n}^{a b}\right)$

The parameter $\sigma_{i n}^{N N}$ has been adjusted to fit the experimental inelastic $p p$ cross section for non-single-diffractive (NSD) events. The use of the data on the NSD $p p$ events is reasonable because the diffractive events do not contribute to the multiplicity in the midrapidity region that we consider.

We assume isentropic evolution of the QGP fireball. Then the initial entropy rapidity density produced in an $A A$ collision is proportional to the final charged multiplicity pseudorapidity density

$d S / d y=C d N_{c h} / d \eta$

where $C \approx 7.67$ [25]. For this reason in our calculations we consider the soft and the hard sources of the entropy as direct sources of the multiplicity density. We will consider the charged multiplicity density $d N_{c h} / d \eta$ at the central pseudorapidity $\eta=0$ defined as $N_{c h}$ in the unit pseudorapidity window $|\eta|<0.5$. We assume that the sources generated in all possible collisions of the bare constituents (i.e., for $N N, M N$, and $M M$ collisions) have the same intensity. This approximation is supported by the calculations within the quark-gluon string model [20,21] which show that the difference between the midrapidity multiplicity density generated in $N N, M N$ and $M M$ interactions is small.

We model the fluctuations of the charged particle density generated by the sources by the Gamma distribution

$\Gamma(n,\langle n\rangle)=\left(\frac{n}{\langle n\rangle}\right)^{\kappa-1} \frac{\kappa^{\kappa} \exp [-n \kappa /\langle n\rangle]}{\langle n\rangle \Gamma(\kappa)}$,

which is widely used in the MCG simulations. For each soft source corresponding to a wounded constituent the contribution to the multiplicity density is given by $(1-\alpha) \Gamma / 2$, and for a hard source from a binary collision it is simply $\Gamma$. However, for each pair of wounded particles the probability 
of a hard binary collision is suppressed by $\alpha$. The parameters $\langle n\rangle$ and $\kappa$ have been adjusted to reproduce the experimental $p p$ data on the mean charged multiplicity and its variance in the unit pseudorapidity window $|\eta|<0.5$, and the value of $\alpha$ has been fitted from the data on $\mathrm{Pb}+\mathrm{Pb}$ collisions (see below).

For calculations of the multiplicity density $d N_{c h} / d \eta$ one can use the approximation of the point-like sources. But the smearing of the sources may be important in calculations of the initial anisotropy coefficients $\varepsilon_{n}$ of the QGP fireball, which in terms of the spacial entropy distribution $\rho_{s}=d S / d y d \rho \operatorname{read}[26,27]$

$\varepsilon_{n}=\frac{\left|\int d \rho \rho^{n} e^{i n \phi} \rho_{s}(\boldsymbol{\rho})\right|}{\int d \rho \rho^{n} \rho_{s}(\boldsymbol{\rho})}$,

where the transverse vectors $\rho$ are calculated in the c.m. frame, i.e., $\int d \rho \rho \rho_{s}(\boldsymbol{\rho})=0$. To model the smearing of the sources we use a Gaussian source distribution

$\exp \left(-\rho^{2} / \sigma^{2}\right) / \pi \sigma^{2}$

We perform calculations for $\sigma=0.7$ and $0.4 \mathrm{fm}$. The results for the anisotropy coefficients become sensitive to $\sigma$ only for very peripheral $A A$ collisions.

We perform calculations for the Woods-Saxon nuclear distributions with the hard-core repulsion. Following to Ref. [9], we take for the hard-core radius $d=0.9 \mathrm{fm}$. For the one-body Woods-Saxon distributions for ${ }^{129} \mathrm{Xe}$ we use the $\theta$-dependent nuclear density

$$
\begin{aligned}
\rho_{A}(r, \theta) & =\frac{\rho_{0}}{1+\exp \left[\left(r-R_{A}(\theta) / a\right]\right.}, \\
R_{A}(\theta) & =R\left[1+\beta_{2} Y_{20}(\theta)+\beta_{4} Y_{40}(\theta)\right]
\end{aligned}
$$

with $Y_{20}$ and $Y_{40}$ the spherical harmonics, with $\beta_{2}=0.162$, and $\beta_{4}=-0.003$ [28]. For the ${ }^{208} \mathrm{~Pb}$ nucleus we use the ordinary spherically symmetric Woods-Saxon formula with a $\theta$-independent $\left(\beta_{02}=\beta_{04}=0\right.$ ) radius $R_{A}$. For the nucleus radii we use the formula $R=\left(1.1 A^{1 / 3}-0.656 / A^{1 / 3}\right) \mathrm{fm}$, and take $a=0.459 \mathrm{fm}$ borrowed from Ref. [9]. To understand the role of the prolate shape of the Xe nucleus, we also perform calculations for $\mathrm{Xe}+\mathrm{Xe}$ collisions using the spherically symmetric Woods-Saxon formula. As will be seen from our results the prolate shape of the Xe nucleus increases noticeably the ellipticity $\varepsilon_{2}$ at small centralities.

\section{Results}

The direct $p p$ data on the charged multiplicity and inelastic cross section at $\sqrt{s}=5.02$ and $5.44 \mathrm{TeV}$ are absent. We obtained $d N_{c h} / d \eta$ for these energies with the help of the power law interpolation $d N_{c h} / d \eta \propto s^{\delta}$ between the ALICE data [29] at $\sqrt{s}=2.76 \mathrm{TeV}\left(d N_{c h} / d \eta \approx 4.63\right)$ and at $\sqrt{s}=$ $7 \mathrm{TeV}\left(d N_{c h} / d \eta=\approx 5.74\right)$ for the charged multiplicity in NSD events. It gives $d N_{c h} / d \eta[\sqrt{s}=5.02,5.44 \mathrm{TeV}] \approx$ $[5.32,5.42]$. We used a similar procedure to obtain the NSD $p p$ inelastic cross sections at $\sqrt{s}=5.02$ and $5.44 \mathrm{TeV}$ from the ALICE [30] results for $\sqrt{s}=2.76 \mathrm{TeV}\left(\sigma_{i n}^{N S D} \approx 50.24\right.$ $\mathrm{mb})$ and $7 \mathrm{TeV}\left(\sigma_{i n}^{N S D} \approx 58.56 \mathrm{mb}\right)$. We obtained the values: $\sigma_{i n}^{p p}[\sqrt{s}=5.02,5.44 \mathrm{TeV}] \approx[54.44,56.18] \mathrm{mb}$.

Since the difference in the energy between $\mathrm{Xe}+\mathrm{Xe}$ collisions at $\sqrt{s}=5.44 \mathrm{TeV}$ and $\mathrm{Pb}+\mathrm{Pb}$ collisions at $\sqrt{s}=5.02$ $\mathrm{TeV}$ is relatively small, we use the same fraction of the binary collisions $\alpha$ for $\sqrt{s}=5.02$ and $5.44 \mathrm{TeV}$. We determine $\alpha$ from fits to the ALICE [31] data on the centrality dependence of the midrapidity charged multiplicity density $N_{c h}$ at $|\eta|<0.5$ in $\mathrm{Pb}+\mathrm{Pb}$ collisions $\sqrt{s}=5.02 \mathrm{TeV}$. As in Ref. [12], we use a two step procedure. First, we fitted $\langle n\rangle$ and $\kappa$ for a broad set of $\alpha$ to the $N_{c h}$ in $p p$ collisions imposing the condition $N_{c h} / D=1$, which is well satisfied for $|\eta|<0.5$ window $[29,32]$. Then, we used the values of $\langle n\rangle$ and $\kappa$ to fit the parameter $\alpha$ from the $\mathrm{Pb}+\mathrm{Pb}$ data. This procedure gives $\alpha \approx 0.09$ and $\alpha \approx 0.13$ for the scenarios with and without meson cloud, respectively. The parameters of the Gamma distribution (6) obtained from the fit with meson cloud to the $p p$ data for the above optimal value $\alpha=0.09$ read: $\langle n\rangle \approx 4.69(4.8), \kappa \approx 0.516(0.524)$ for $\sqrt{s}=5.02(5.44) \mathrm{TeV}$. For the scenario without meson cloud for the optimal value $\alpha=0.13$ we obtained $\kappa \approx 0.56(0.56)$ for $\sqrt{s}=5.02(5.44) \mathrm{TeV}$, (in the version without meson cloud the value of $\langle n\rangle$ is simply equal to the experimental $N_{c h}$ for $p p$ collisions).

In Fig. 1 we show the results of our fit to the ALICE data on the centrality dependence of the midrapidity charged multiplicity density in $\mathrm{Pb}+\mathrm{Pb}$ collisions at $\sqrt{s}=5.02 \mathrm{TeV}$ [31] obtained by Monte Carlo generation of $\sim 2 \cdot 10^{6}$ events for the scenarios with and without meson cloud. The scenario with meson cloud gives somewhat better agreement with the data $\left(\chi^{2} /\right.$ d.p $\left.\approx 0.1\right)$ as compared to the version without meson cloud $\left(\chi^{2} /\right.$ d.p $\left.\approx 0.3\right)$.

In Fig. 2 we show our predictions for centrality dependence of the charged multiplicity in $\mathrm{Xe}+\mathrm{Xe}$ collisions at $\sqrt{s}=5.44 \mathrm{TeV}$ obtained with and without meson cloud. The difference between two version is relatively small. For intermediate centrality region the meson cloud increases $d N_{c h} / d \eta$ by $\sim 5 \%$. For the $0-5 \%$ centrality $d N_{c h} / d \eta \approx$ 1149 and 1134 for the versions with and without meson cloud, respectively. It is smaller by $\sim 6-7 \%$ than the charged multiplicity density obtained in Ref. [4]. From Figs. 1 to 2 one sees that as compared to $\mathrm{Pb}+\mathrm{Pb}$ collisions at $\sqrt{s}=5.02$ $\mathrm{TeV}$ for Xe $+\mathrm{Xe} 0-5 \%$ central collisions at $\sqrt{s}=5.44 \mathrm{TeV}$, $d N_{c h} / d \eta$ becomes smaller by a factor of $\sim 1.7$. That corresponds to decrease of the initial QGP temperature by a factor of $\sim$ 1.2. A remark is in order here. The curves in Fig. 2 are obtained under assumption of an isentropic flow. But we 

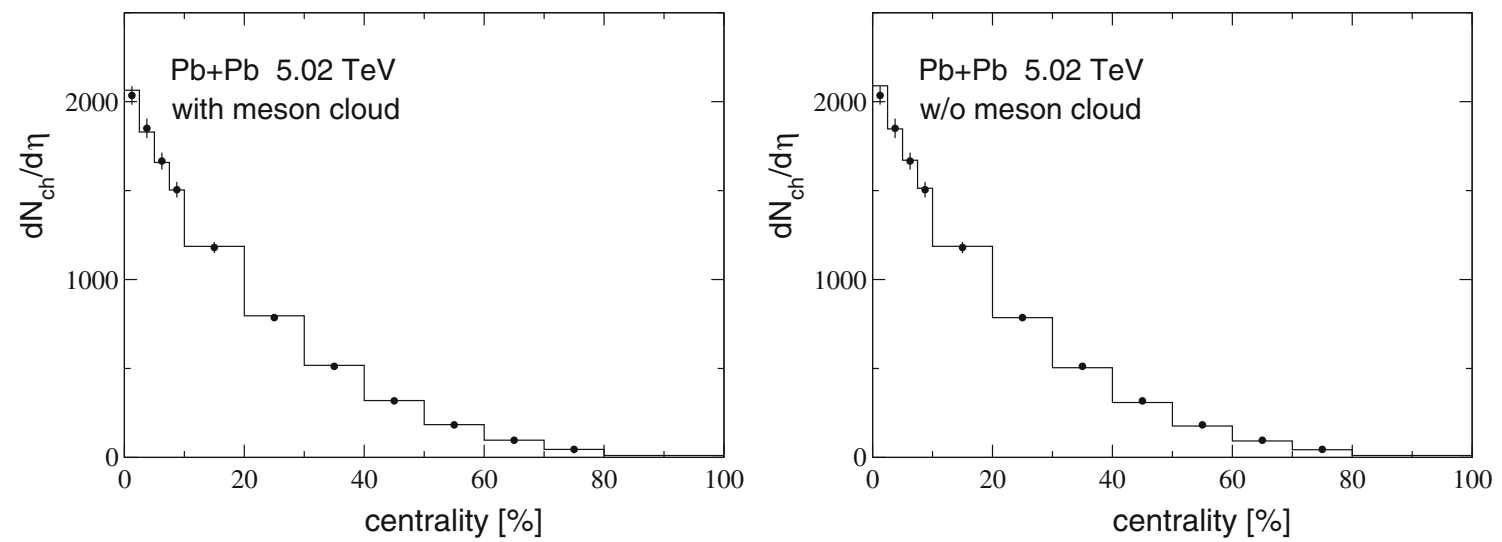

Fig. 1 Centrality dependence of midrapidity $d N_{c h} / d \eta$ for $\mathrm{Pb}+\mathrm{Pb}$ collisions at $\sqrt{s}=5.02 \mathrm{TeV}$. Left: MCG simulation for the scenario with meson cloud at $\alpha=0.09$. Right: MCG simulation for the scenario without meson cloud at $\alpha=0.13$. Data are from ALICE [31]

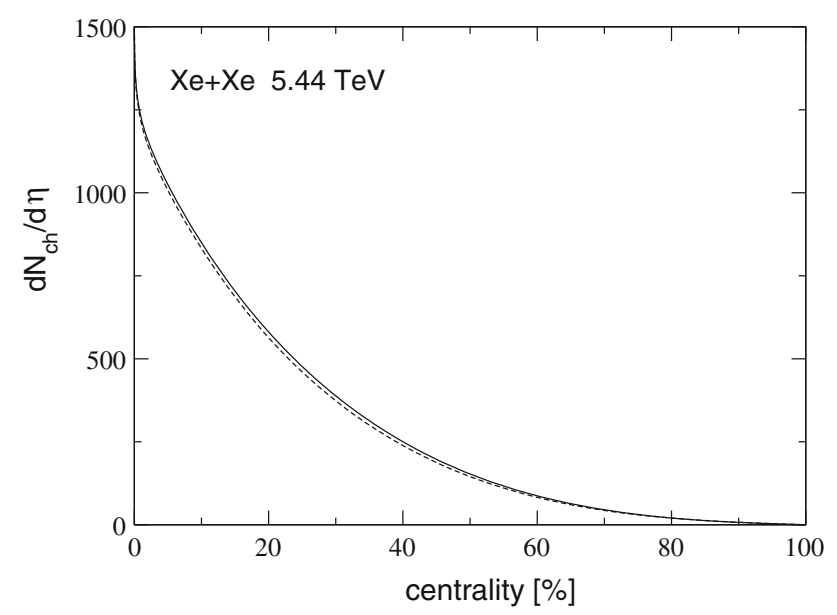

Fig. 2 Centrality dependence of midrapidity $d N_{c h} / d \eta$ for $\mathrm{Xe}+\mathrm{Xe}$ collisions at $\sqrt{s}=5.44 \mathrm{TeV}$ obtained for the scenarios with (solid) meson cloud at $\alpha=0.09$ and without (dashed) meson cloud at $\alpha=0.13$

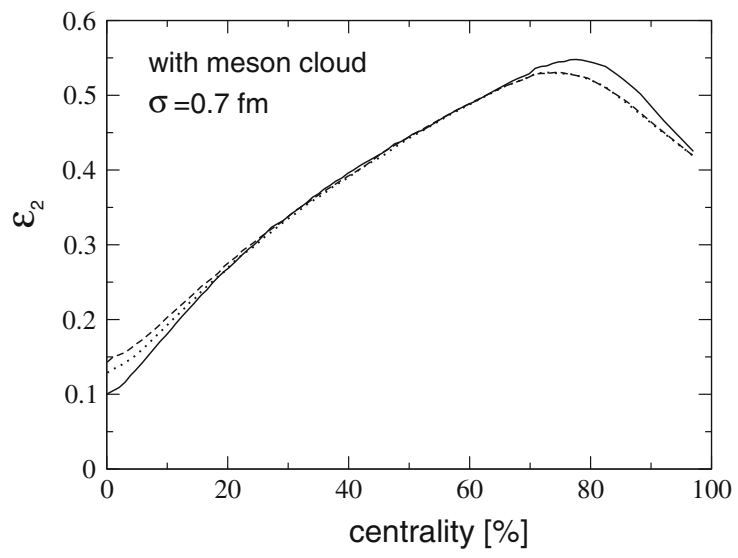

Fig. 3 Centrality dependence of the rms $\varepsilon_{2}$ for the Gaussian source distribution (8) for $\sigma=0.7 \mathrm{fm}$ for $\mathrm{Pb}+\mathrm{Pb}$ collisions at $\sqrt{s}=5.02 \mathrm{TeV}$ (solid) and $\mathrm{Xe}+\mathrm{Xe}$ collisions at $\sqrt{s}=5.44 \mathrm{TeV}$ for the $\theta$-dependent fitted the parameters of the model from the data on $\mathrm{Pb}+$ $\mathrm{Pb}$ collisions also ignoring the non-isentropic effects. Since these effects are weak, possible errors in the extrapolation of the results from $\mathrm{Pb}+\mathrm{Pb}$ to $\mathrm{Xe}+\mathrm{Xe}$ collisions should be small.

In Figs. 3 and 4 we present the results for the rms $\varepsilon_{2}$ (the $\operatorname{rms} \varepsilon_{n}$ is often denoted $\varepsilon_{n}\{2\}$, for clarity, we omit $\{2\}$ ) versus centrality for $\mathrm{Pb}+\mathrm{Pb}$ at $\sqrt{s}=5.02 \mathrm{TeV}$ and $\mathrm{Xe}$ $+\mathrm{Xe}$ at $\sqrt{s}=5.44 \mathrm{TeV}$ for two version of the model. The anisotropy coefficients depend on the smearing parameter $\sigma$ in (8). We present the results for two values of the Gaussian width of the sources $\sigma=0.7$ and $0.4 \mathrm{fm}$. From Figs. 3 to 4 one sees that for small centralities the results with and without meson cloud are close to each other. For intermediate centralities the version with meson cloud gives a little smaller $\varepsilon_{2}$. For very peripheral collisions with centrality $\gtrsim 80 \%$ the model without meson cloud gives bigger $\varepsilon_{2}$. From comparing the results in Figs. 3 and 4 one can

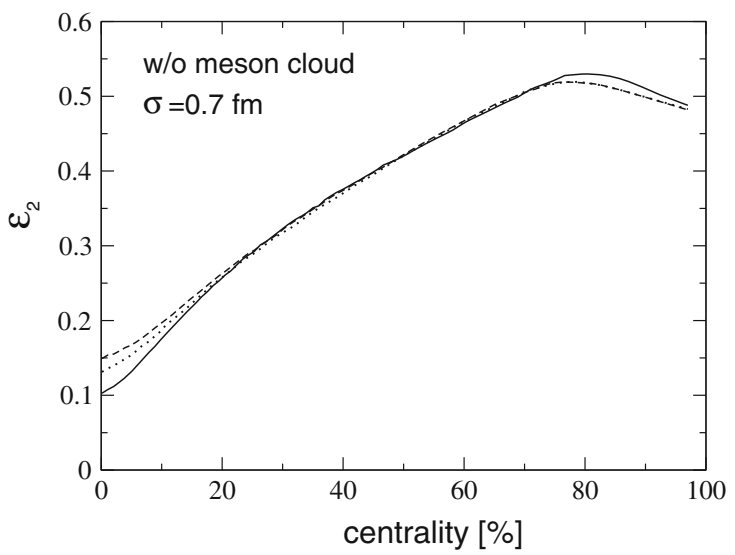

(dashed) and symmetric Woods-Saxon distribution (dotted). Left: the version with meson cloud at $\alpha=0.09$. Right: the version without meson cloud at $\alpha=0.13$ 

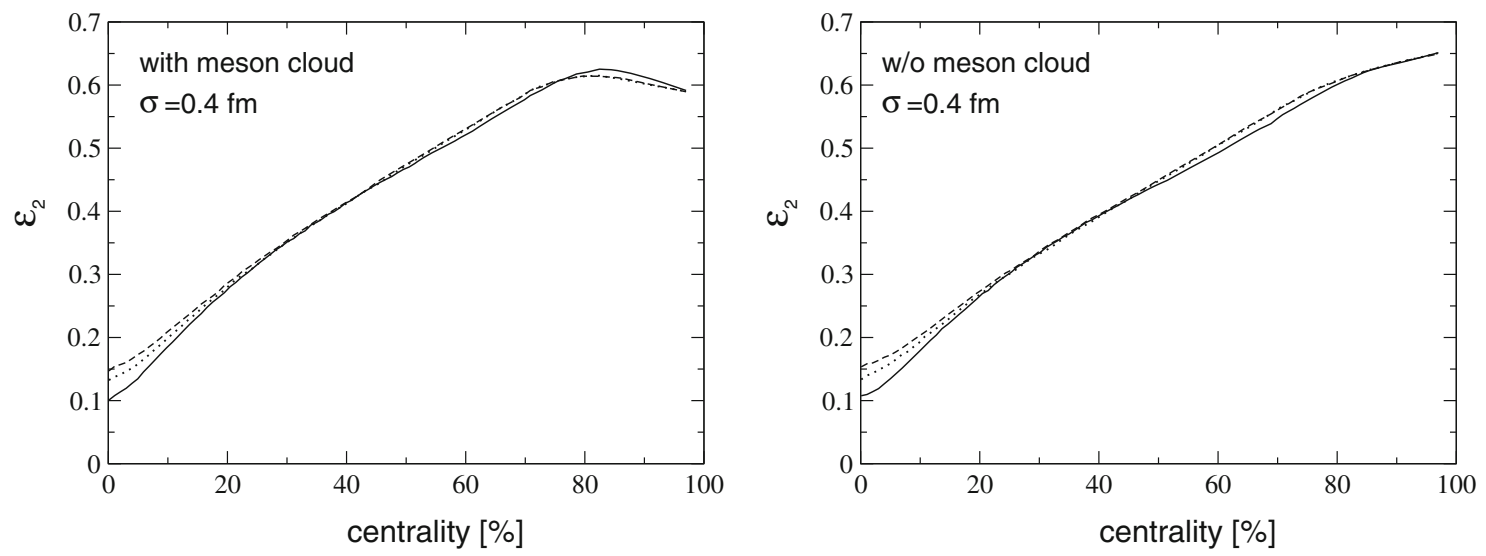

Fig. 4 Same as in Fig. 3 but for $\sigma=0.4 \mathrm{fm}$
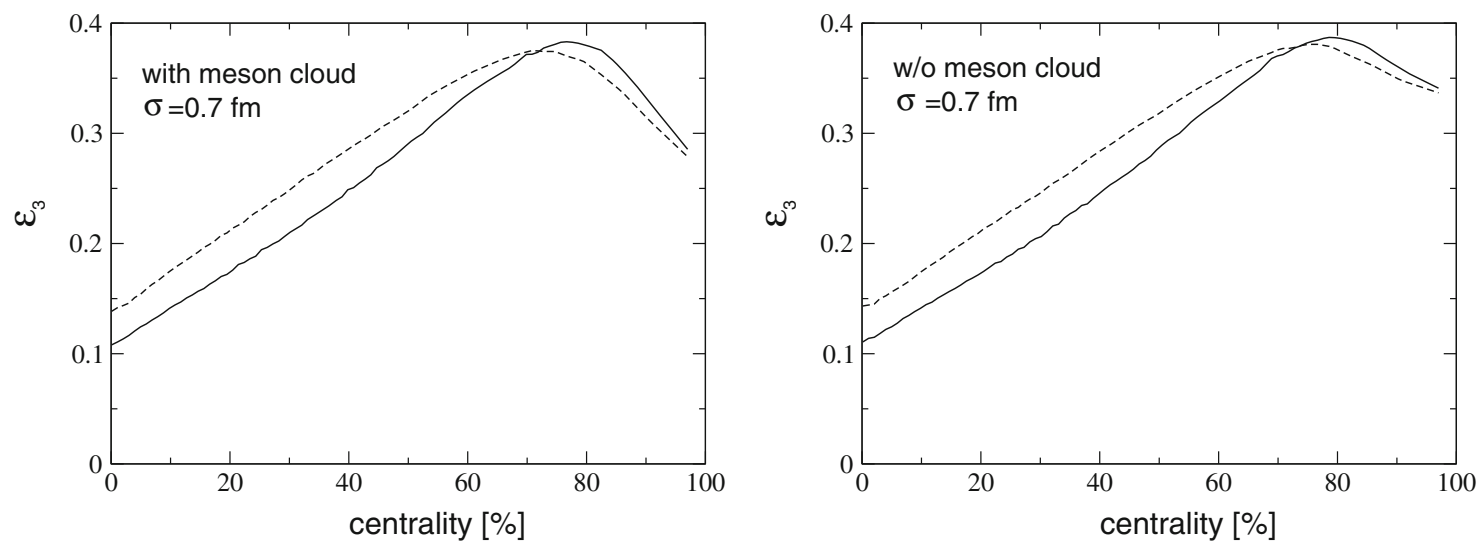

Fig. 5 Centrality dependence of the rms $\varepsilon_{3}$ for the Gaussian source distribution (8) for $\sigma=0.7 \mathrm{fm}$ for $\mathrm{Pb}+\mathrm{Pb}$ collisions at $\sqrt{s}=5.02 \mathrm{TeV}$ (solid) and $\mathrm{Xe}+\mathrm{Xe}$ collisions at $\sqrt{s}=5.44 \mathrm{TeV}$ for the $\theta$-dependent

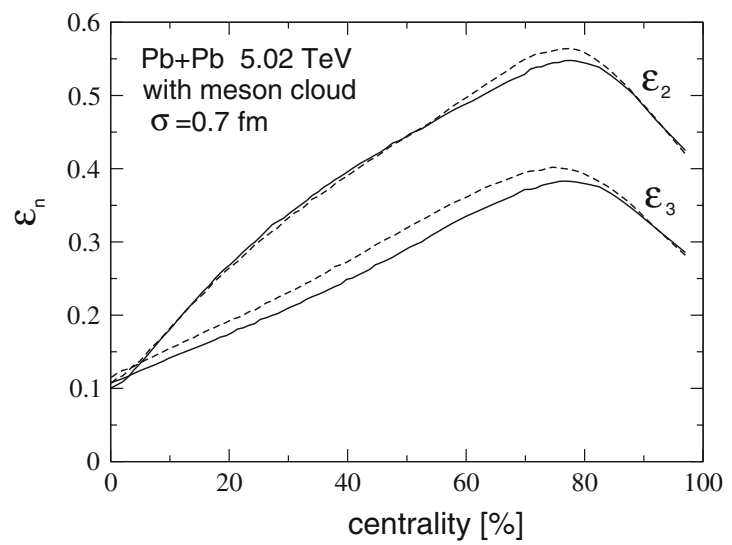

Fig. 6 Centrality dependence of the rms $\varepsilon_{2,3}$ for the version with meson cloud with the Gaussian source distribution (8) for $\sigma=0.7 \mathrm{fm}$ in $\mathrm{Pb}$ $+\mathrm{Pb}$ collisions at $\sqrt{s}=5.02 \mathrm{TeV}$ (left) and in $\mathrm{Xe}+\mathrm{Xe}$ collisions at

see that the value of the smearing width becomes important at $c \gtrsim 30-40 \%$, where the eccentricity grows with decreasing $\sigma$. From Figs. 3 to 4 one sees that for the most

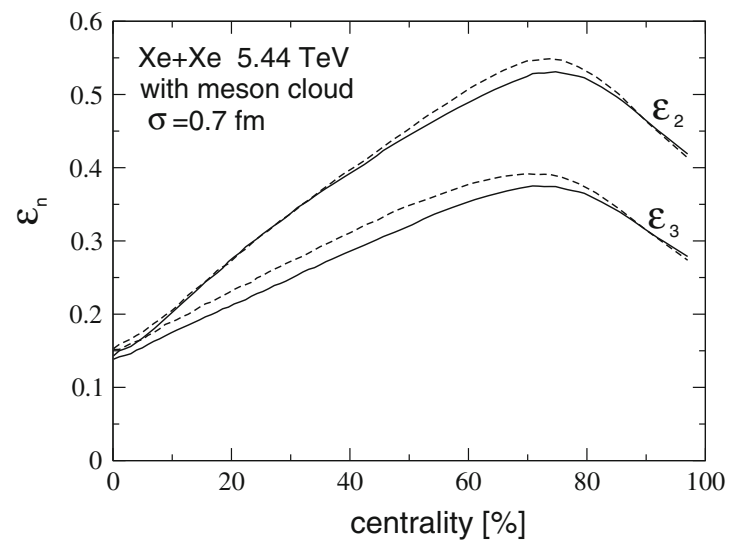

$\sqrt{s}=5.44 \mathrm{TeV}$ (right) for the $\theta$-dependent Woods-Saxon distribution with (solid) and without (dashed) the hard-core repulsion

central collisions $c \lesssim 5 \% \varepsilon_{s}(\mathrm{Xe}) / \varepsilon_{2}(\mathrm{~Pb}) \sim 1.3-1.4$, and $\varepsilon_{s}(\mathrm{Xe}) / \varepsilon_{2}(\mathrm{~Pb})$ becomes close to unity at $c \gtrsim 30 \%$. For very peripheral collisions with $c \gtrsim 75 \% \varepsilon_{2}(\mathrm{Xe})$ becomes a little 


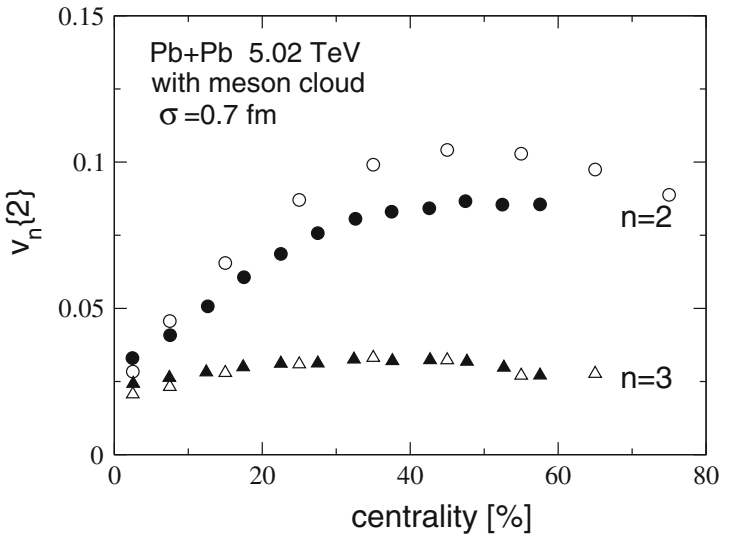

Fig. 7 Centrality dependence of $v_{2}\{2\}$ (filled circles) and $v_{3}\{2\}$ (filled triangles) for $\mathrm{Pb}+\mathrm{Pb}$ collisions at $\sqrt{s}=5.02 \mathrm{TeV}$ obtained from $\varepsilon_{2,3}$ with meson cloud for $\sigma=0.7$ (left) and $0.4 \mathrm{fm}$ (right) with the help of

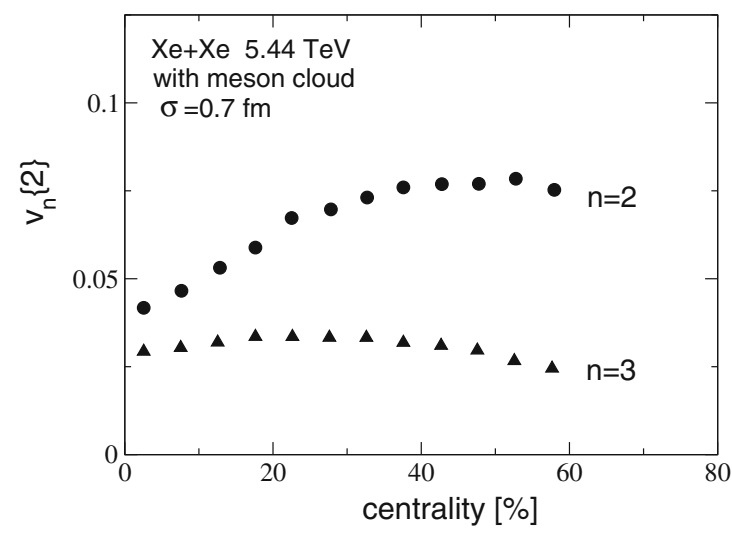

Fig. 8 Same as in Fig. 7 but for $\mathrm{Xe}+\mathrm{Xe}$ collisions at $\sqrt{s}=5.44 \mathrm{TeV}$

smaller than $\varepsilon_{2}(\mathrm{~Pb})$. From Figs. 3 to 4 , by comparing the curves for $\mathrm{Xe}+\mathrm{Xe}$ collisions obtained for the asymmetric and symmetric Woods-Saxon distribution, one can see that the effect of the prolate shape of the Xe nucleus on the eccentricity becomes important at $c \lesssim 20 \%$. For most central collisions the asymmetric Woods-Saxon distribution increases the eccentricity by $\sim 12 \%$. This is due to the highly overlapping body-body collisions of the nucleus ellipsoids, that lead naturally to an asymmetric entropy deposition.

In Fig. 5 we present the results for the rms triangularity $\varepsilon_{3}$ for $\sigma=0.7 \mathrm{fm}$. One can see that both for $\mathrm{Pb}+\mathrm{Pb}$ and $\mathrm{Xe}+$ $\mathrm{Xe}$ collisions the triangularity for the versions with and without meson cloud are very similar for small and intermediate centralities. For very peripheral collisions $c \gtrsim 80 \% \varepsilon_{3}$ for the version without meson cloud becomes bigger. Our calculations show that for $\mathrm{Xe}+\mathrm{Xe}$ collisions the effect of the prolate form of the $\mathrm{Xe}$ nucleus is very small. For this reason we do not plot in Fig. 5 the results for Xe + Xe collisions for the symmetric Woods-Saxon distribution. From Fig. 5 one sees that $\varepsilon_{3}(\mathrm{Xe}) / \varepsilon_{3}(\mathrm{~Pb}) \sim 1.3$ for most central collisions, and decreases to unity at $c \sim 70 \%$.

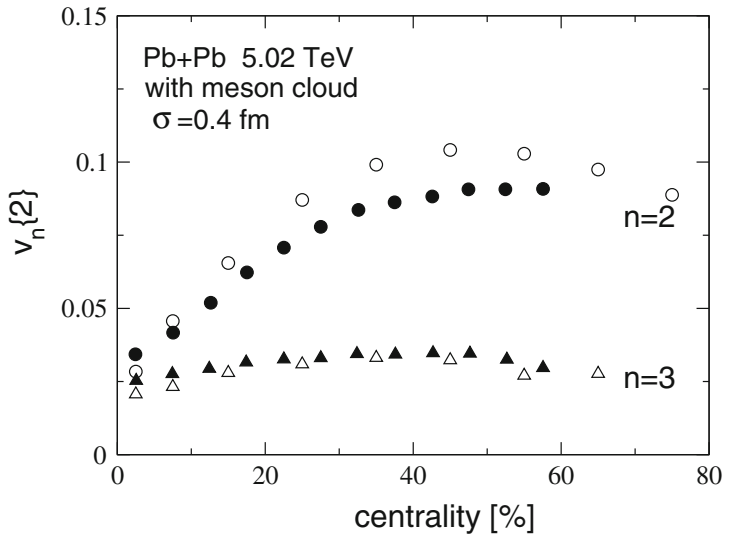

the linear response approximation (see text for explanations) using the results of the hydrodynamic simulations of Ref. [3]. Open circles and triangles are $v_{2,3}\{2\}$ from ALICE [41]

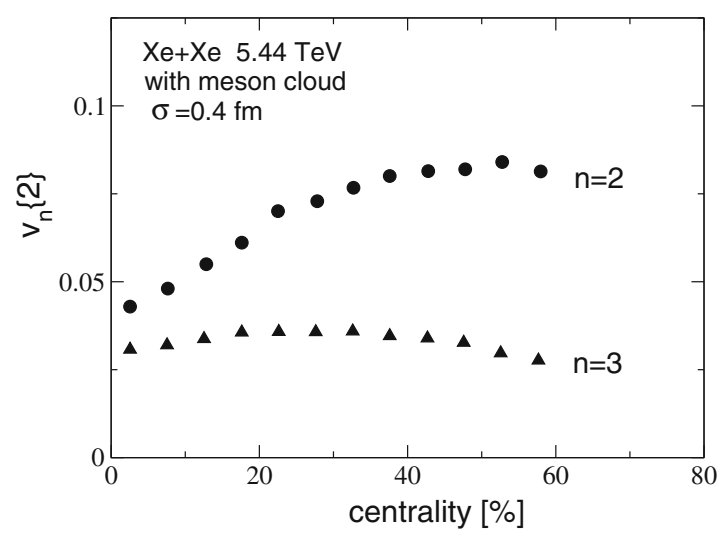

The results shown in Figs. 1, 2, 3, 4 and 5 have been obtained for the Monte-Carlo sampling with the nuclear distribution with the hard-core radius $d=0.9 \mathrm{fm}$. It is evident that the presence of the hard-core repulsion should reduce the fluctuations of the nuclear matter density in the colliding nuclei, that can suppress the effect of the fluctuations in the initial entropy deposition. To study the role of the hard-core repulsion we also performed the calculations for $d=0$. In this case we also use the parameters of the Woods-Saxon distribution from the analysis [9]: $R=\left(1.12 A^{1 / 3}-0.86 / A^{1 / 3}\right)$ $\mathrm{fm}$, and $a=0.54 \mathrm{fm}$. Calculations in this version show that the effect of the hard-core repulsion on the centrality dependence of the charged multiplicity density is small. But the effect is stronger for the anisotropy coefficients. It is illustrated in Fig. 6 where we plot $\varepsilon_{2,3}$ versus centrality obtained with (solid) and without (dashed) the hard-core repulsion for the version with meson cloud at $\sigma=0.7 \mathrm{fm}$. From Fig. 6 one sees that the effect of the hard-core is bigger for the purely fluctuation-driven quantity $\varepsilon_{3}$. At $c \lesssim 70 \%$ the hard-core reduces $\varepsilon_{3}$ by $\sim 10 \%$ both for $\mathrm{Pb}+\mathrm{Pb}$ and $\mathrm{Xe}+\mathrm{Xe}$ collisions. For the eccentricity $\varepsilon_{2}$ the effect of the hard-core is 
noticeable for small centralities. In this region $\varepsilon_{2}$ is also dominated by fluctuations, and the hard-core repulsion reduces $\varepsilon_{2}$ by $\sim 10 \%$ both for $\mathrm{Pb}+\mathrm{Pb}$ and $\mathrm{Xe}+\mathrm{Xe}$ collisions.

Our results for the initial $\varepsilon_{2,3}$ for $\mathrm{Pb}+\mathrm{Pb}$ and $\mathrm{Xe}+\mathrm{Xe}$ collisions do not differ strongly from that of Refs. [3,4]. The analysis [3] is based on the TRENTO Monte-Carlo model $[33,34]$, and [4] is based on the so called EKRT [35] model with mini-jet parton production. For intermediate centralities $c \sim 50 \%$ our $\varepsilon_{2}$ for $\sigma=0.7 \mathrm{fm}$ is smaller than that of Refs. [3,4] by $\sim 10-15 \%$. But for small centralities our predictions for $\varepsilon_{2,3}$ are somewhat bigger. Note that in Refs. [3,4] the calculations for $\mathrm{Xe}+\mathrm{Xe}$ collisions have been performed only with the symmetric Woods-Saxon nuclear distribution. For this reason the grows of the ratio $\varepsilon_{2}(\mathrm{Xe}) / \varepsilon_{2}(\mathrm{~Pb})$ at $c \rightarrow 0$ in Refs. $[3,4]$ is not so strong as in our results.

The event by event hydrodynamic modeling of $A A$ collisions shows that, except for very peripheral collisions, to a good approximation $v_{n} \approx k_{n} \varepsilon_{n}$ for $n=2,3$ [36-39] (for a comprehensive review, see, e.g., Ref. [40]). This linear response approximation works better for $v_{2}[38,39]$. In the present paper we do not perform the hydrodynamic simulation of $\mathrm{Pb}+\mathrm{Pb}$ and $\mathrm{Xe}+\mathrm{Xe}$ collisions that are necessary for accurate calculations of $v_{n}$. To obtain predictions for the flow coefficients via our MCG results for $\varepsilon_{n}$ we use the above linear response relation with $k_{2,3}$ defined via the ratio of $v_{2,3}\{2\}$ to rms $\varepsilon_{2,3}$ obtained for $\mathrm{Pb}+\mathrm{Pb}$ collisions at $\sqrt{s}=5.02 \mathrm{TeV}$ and for $\mathrm{Xe}+\mathrm{Xe}$ collisions at $\sqrt{s}=5.44$ $\mathrm{TeV}$ in the hydrodynamic calculations with the shear viscosity over entropy ratio $\eta / s=0.047$ in Ref. [3]. ${ }^{1}$ In Fig. 7 we compare $v_{2,3}\{2\}$ obtained in this way for $\mathrm{Pb}+\mathrm{Pb}$ collisions at $\sqrt{s}=5.02 \mathrm{TeV}$ with the results from ALICE [41]. We used our anisotropy coefficients $\varepsilon_{2,3}$ obtained with meson cloud for the Woods-Saxon distribution with the hard-core repulsion for the smearing width $\sigma=0.7$ and $0.4 \mathrm{fm}$. From Fig. 7 one sees that for $n=2$ the theoretical results underestimate the data by $\sim 10-20 \%$ at $c \sim 30-60 \%$, but for $n=3$ the agreement is quite reasonable. The theoretical predictions for $v_{2,3}\{2\}$ in $\mathrm{Xe}+\mathrm{Xe}$ collisions at $\sqrt{s}=5.44 \mathrm{TeV}$ are shown in Fig. 8. The relation between our predictions for $v_{n}\{2\}$ to that of Ref. [3] similar to the situation with predictions for $\varepsilon_{n}$. For most central collisions, due to bigger values of $\varepsilon_{2,3}$, we predict somewhat bigger $v_{2,3}\{2\}$ (by $\sim 5 \%$ for $\sigma=0.7$ $\mathrm{fm}$ and by $\sim 10 \%$ for $\sigma=0.4 \mathrm{fm}$ ).

The predictions in Figs. 7 and 8 correspond to the version with the hard-core repulsions. From the curves in Fig. 6 one sees that the hard-core repulsion reduces the initial anisotropy coefficients due some reduction of the the nuclear density fluctuations. However, one should bear in mind that

\footnotetext{
${ }^{1}$ In principle, it is clear that this procedure should be quite accurate even beyond the linear response picture (say, when the cubic terms become important [39]) because the difference between our predictions for $\varepsilon_{2,3}$ and that of Ref. [3] is not very large.
}

the dynamical effects, which can affect the long range fluctuations of the nuclear density, are completely ignored in our Monte-Carlo scheme. For this reason the predictions for the initial anisotropy coefficients may be questioned (especially for the fluctuation driven quantity $\varepsilon_{3}$ ). Indeed, it is well known $[42,43]$ that the dynamical long range fluctuations of the nuclear matter in heavy nuclei are dominated by the giant resonances, e.g., by the giant dipole, monopole, and quadrupole resonances. In Ref. [44] we have demonstrated that for the dipole mode of the ${ }^{208} \mathrm{~Pb}$ nucleus the classical treatment based on the Monte-Carlo simulation with the Woods-Saxon nuclear density overestimates the fluctuations of the dipole moment squared by a factor of $\sim 5$. The situation with the dynamical quantum effects for the monopole and quadrupole collective modes, that potentially may also be important, remains unclear. It would be of great interest to study the role of the dynamical quantum effects due to the giant resonances on the fluctuations of the entropy deposition in $A A$ collisions (of course, these effects may also be important for calculations in other approaches, say, in the EKRT model [35], TRENTO model [34], or in the color glass condensate scheme $[45,46])$. We leave this for future work.

\section{Conclusions}

We have studied the centrality dependence of the charged midrapidity multiplicity density in $\mathrm{Pb}+\mathrm{Pb}$ collisions at $\sqrt{s}=5.02 \mathrm{TeV}$ and in $\mathrm{Xe}+\mathrm{Xe}$ collisions at $\sqrt{s}=5.44$ $\mathrm{TeV}$ within the MCG model with and without meson cloud developed in Ref. [12]. The parameters of the model have been fixed to the ALICE data [31] on $d N_{c h} / d \eta$ in $\mathrm{Pb}+$ $\mathrm{Pb}$ collisions. We obtained the fraction of the binary collisions $\alpha \approx 0.09(0.13)$ with (without) meson cloud. With these parameters we give predictions for future LHC data on $\mathrm{Xe}+\mathrm{Xe}$ collisions at $\sqrt{s}=5.44 \mathrm{TeV}$. We find that the effect of the meson cloud on the $d N_{c h} / d \eta$ is relatively small. For Xe + Xe collisions the meson cloud increases $d N_{c h} / d \eta$ by $\sim 5 \%$ in the intermediate centrality region. For the $0-5 \%$ centrality bin we obtained $d N_{c h} / d \eta \approx 1149$ and 1134 with and without meson cloud, respectively. As compared to $\mathrm{Pb}$ $+\mathrm{Pb}$ collisions at $\sqrt{s}=5.02 \mathrm{TeV}$ for $\mathrm{Xe}+\mathrm{Xe} 0-5 \%$ central collisions at $\sqrt{s}=5.44 d N_{c h} / d \eta$ becomes smaller by a factor of $\sim 1.7$. It corresponds to decrease of the initial QGP temperature by a factor of $\sim 1.2$.

Both for $\mathrm{Pb}+\mathrm{Pb}$ and $\mathrm{Xe}+\mathrm{Xe}$ collisions we do not find a significant effect of the meson cloud on the $\varepsilon_{2,3}$ at $c \lesssim$ $70 \%$. But the meson cloud reduces $\varepsilon_{2,3}$ for very peripheral collisions. We find that the ratio of the eccentricity in $\mathrm{Xe}+$ $\mathrm{Xe}$ collisions to that for $\mathrm{Pb}+\mathrm{Pb}$ collisions is close to unity at $c \gtrsim 20 \%$, but it becomes bigger than unity at $c \lesssim 20 \%$. We obtained $\varepsilon_{2}(\mathrm{Xe}) / \varepsilon_{2}(\mathrm{~Pb}) \sim 1.45$ for most central collisions $(c \lesssim 1 \%)$. We predict a noticeable increase of the eccentricity 
in $\mathrm{Xe}+\mathrm{Xe}$ collisions at small centralities due to the prolate shape of the Xe nucleus. This effect gives $\sim 50 \%$ to the difference between the eccentricity in most central $\mathrm{Xe}+\mathrm{Xe}$ and $\mathrm{Pb}+\mathrm{Pb}$ collisions.

We find that at $c \lesssim 70 \%$ the triangularity in $\mathrm{Xe}+\mathrm{Xe}$ collisions is bigger than in $\mathrm{Pb}+\mathrm{Pb}$ collisions. We obtain $\varepsilon_{3}(\mathrm{Xe}) / \varepsilon_{3}(\mathrm{~Pb}) \sim 1.3$ at $c \lesssim 1 \%$ and $\varepsilon_{3}(\mathrm{Xe}) / \varepsilon_{3}(\mathrm{~Pb}) \sim 1.1$ at $c \sim 50 \%$. We have investigated the effect of the hardcore repulsion in the Monte-Carlo sampling of the nuclear distributions. We found that the hard-core repulsion gives a relatively small effect on the charged multiplicity density. But its effect is sizeable for the anisotropy coefficients $\varepsilon_{2,3}$. For $\varepsilon_{2}$ the effect of the hard-core is noticeable for small centralities where $\varepsilon_{2}$ is dominated by fluctuations. For most central collisions the hard-core repulsion reduces $\varepsilon_{2}$ by $\sim 10 \%$ both for $\mathrm{Pb}+\mathrm{Pb}$ and $\mathrm{Xe}+\mathrm{Xe}$ collisions. The triangularity $\varepsilon_{3}$ is reduced by the hard-core repulsion by $\sim 10 \%$ at $c \lesssim 70 \%$ both for for $\mathrm{Pb}+\mathrm{Pb}$ and $\mathrm{Xe}+\mathrm{Xe}$ collisions.

To obtain predictions for the flow coefficients via our MCG results for $\varepsilon_{n}$ we have used the linear response relation $v_{n} \approx k_{n} \varepsilon_{n}$ with $k_{n}$ defined via the ratio of $v_{2,3}\{2\}$ to $\mathrm{rms}$ $\varepsilon_{2,3}$ obtained in the recent hydrodynamic analysis [3]. The results for $v_{2,3}\{2\}$ obtained in this way for $\mathrm{Pb}+\mathrm{Pb}$ collisions at $\sqrt{s}=5.02 \mathrm{TeV}$ are in reasonable agreement with the data from ALICE [41].

Open Access This article is distributed under the terms of the Creative Commons Attribution 4.0 International License (http://creativecomm ons.org/licenses/by/4.0/), which permits unrestricted use, distribution, and reproduction in any medium, provided you give appropriate credit to the original author(s) and the source, provide a link to the Creative Commons license, and indicate if changes were made. Funded by $\mathrm{SCOAP}^{3}$.

\section{References}

1. P. Huovinen, Int. J. Mod. Phys. E 22, 1330029 (2013). arXiv:1311.1849 (and references therein)

2. R. Derradi de Souza, T. Koide, T. Kodama, Prog. Part. Nucl. Phys. 86, 35 (2016). arXiv: 1506.03863 (and references therein)

3. G. Giacalone, J. Noronha-Hostler, M. Luzum, U. Sao Paulo, J.Y. Ollitrault, Phys. Rev. C 97, 034904 (2018). arXiv:1711.08499

4. K.J. Eskola, H. Niemi, R. Paatelainen, K. Tuominen, Phys. Rev. C 97, 034911 (2018). arXiv:1711.09803

5. U. Heinz, R. Snellings, Ann. Rev. Nucl. Part. Sci. 63, 123 (2013). arXiv: 1301.2826

6. H. Song, S.A. Bass, U. Heinz, T. Hirano, Phys. Rev. C 83, 054910 (2011). arXiv:1101.4638 (Erratum. Phys. Rev. C 86, 059903 (2012))

7. B. Alver, M. Baker, C. Loizides, P. Steinberg. arXiv:0805.4411

8. W. Broniowski, M. Rybczynski, P. Bozek, Comput. Phys. Commun. 180, 69 (2009). arXiv:0710.5731

9. M. Rybczynski, G. Stefanek, W. Broniowski, P. Bozek, Comput. Phys. Commun. 185, 1759 (2014). arXiv: 1310.5475

10. C. Loizides, J. Kamin, D. d'Enterria, Phys. Rev. C 97, 054910 (2018). arXiv:1710.07098
11. B.G. Zakharov, JETP Lett. 104, 6 (2016). arXiv:1605.06012

12. B.G. Zakharov, J. Exp. Theor. Phys. 124, 860 (2017). arXiv: 1611.05825

13. J. Speth, A.W. Thomas, Adv. Nucl. Phys. 24, 83 (1997)

14. A. Bialas, W. Czyz, W. Furmanski, Acta Phys. Pol. B 8, 585 (1977)

15. A. Bialas, W. Czyz, Acta Phys. Pol. B 10, 831 (1979)

16. S. Eremin, S. Voloshin, Phys. Rev. C 67, 064905 (2003). arxiv:nucl-th/0302071

17. P. Bozek, W. Broniowski, M. Rybczynski, Phys. Rev. C 94, 014902 (2016). arXiv: 1604.07697

18. A. Bialas, A. Bzdak, Phys. Rev. C 77, 034908 (2008). arXiv:0707.3720

19. C. Loizides, Phys. Rev. C 94, 024914 (2016). arXiv: 1603.07375

20. A.B. Kaidalov, M.G. Poghosyan, Eur. Phys. J. C 67, 397 (2010). arXiv:0910.2050

21. A. Capella, E.G. Ferreiro, Eur. Phys. J. C 72, 1936 (2012). arXiv: 1110.6839

22. D. Kharzeev, M. Nardi, Phys. Lett. B 507, 121 (2001). arxiv:nucl-th/0012025

23. L. Adamczyk et al., STAR Collaboration, Phys. Rev. Lett. 115, 222301 (2015). arXiv:1505.07812

24. H. Holtmann, A. Szczurek, J. Speth, Nucl. Phys. A 596, 631 (1996). arxiv:hep-ph/9601388

25. B. Müller, K. Rajagopal, Eur. Phys. J. C 43, 15 (2005). arxiv:hep-ph/0502174

26. D. Teaney, L. Yan, Phys. Rev. C 83, 064904 (2011). arXiv: 1010.1876

27. E. Retinskaya, M. Luzum, J.-Y. Ollitrault, Nucl. Phys. A 926, 152 (2014). arXiv:1401.3241

28. P. Möller, A.J. Sierk, T. Ichikawa, H. Sagawa, Atom. Data Nucl. Data Tables 109-110, 1 (2016). arXiv: 1508.06294

29. J. Adam, et al., ALICE Collaboration, Eur. Phys. J. C 77, 33 (2017). arXiv: 1509.07541

30. B. Abelev, et al., ALICE Collaboration, Eur. Phys. J. C 73, 2456 (2013). arXiv: 1208.4968

31. J. Adam, et al., ALICE Collaboration, Phys. Rev. Lett. 116, 222302 (2016). arXiv:1512.06104

32. C. Albajar, et al., UA1 Collaboration, Nucl. Phys. B 335, 261 (1990)

33. J.S. Moreland, J.E. Bernhard, S.A. Bass, Phys. Rev. C 92, 011901 (2015). arXiv:1412.4708

34. J.E. Bernhard, J.S. Moreland, S.A. Bass, Nucl. Phys. A 967, 293 (2017). arXiv: 1704.04462

35. K.J. Eskola, K. Kajantie, P.V. Ruuskanen, K. Tuominen, Nucl. Phys. B 570, 379 (2000). arxiv:hep-ph/9909456

36. H. Song, U.W. Heinz, Phys. Rev. C 78, 024902 (2008). arXiv:0805.1756

37. Z. Qiu, U.W. Heinz, Phys. Rev. C 84, 024911 (2011). arXiv: 1104.0650

38. H. Niemi, G.S. Denicol, H. Holopainen, P. Huovinen, Phys. Rev. C 87, 054901 (2013). arXiv:1212.1008

39. J. Noronha-Hostler, L. Yan, F.G. Gardim, J.-Y. Ollitrault, Phys. Rev. C 93, 014909 (2016). arXiv: 1511.03896

40. L. Yan, Chin. Phys. C 42, 042001 (2018). arXiv:1712.04580

41. J. Adam, et al., ALICE Collaboration, Phys. Rev. Lett. 116, 132302 (2016). arXiv:1602.01119

42. W. Greiner, J.A. Maruhn, Nucl. Models (Springer, Berlin, 1996)

43. S. Kamerdzhiev, J. Speth, G. Tertychny, Phys. Rep. 393, 1 (2004). arxiv:nucl-th/0311058

44. B.G. Zakharov, JETP Lett. 105, 758 (2017). arXiv:1703.04271

45. B. Schenke, P. Tribedy, R. Venugopalan, Phys. Rev. Lett. 108, 252301 (2012). arXiv: 1202.6646

46. B. Schenke, P. Tribedy, R. Venugopalan, Phys. Rev. C 86, 034908 (2012). arXiv:1206.6805 